Notes.

$a$. The chief star of $\mathrm{h} 3556$.
$b$. Position given is that of I Velorum, the comes $50^{\prime \prime}$ distant is double.

c. The comes of the old pair Dunlop 189 .

d. A quadruple star:

$$
\begin{aligned}
& \mathrm{B}=9.5 \mathrm{mag} . \mathbf{1}^{\circ} \mathrm{2}^{\prime \prime} \text { Melbourne } 1878 \\
& \mathrm{C}=\text { New Companion } \\
& \mathrm{D}=\mathrm{h} 4876 .
\end{aligned}
$$

$e$. One night's observation only. f. The chief star of h $5 \mathrm{I} 4 \mathrm{I}$.

g. $v$ Microscopii is $65^{\prime \prime} \mathrm{s} . \mathrm{p}$.

The above new double stars were discovered by $\mathrm{Mr}$. R.T.A. Innes, with the 6.9 in. Equatoreal of the Cape Observatory between the months of April and October 1896 .

The position angles and distances are estimations as the driving of the telescope by the clock is not steady enough to allow of micrometrical measures.

Royal Observatory, Cape of Good Hope, 1896 Oct. 27.

Zusatz. Im Anschluss an das vorstehende Verzeichniss macht Herr R. T. A. Innes d. d. 12. Jan. 1897 noch die folgenden Bemerkungen.

P. III. 19. The duplicity of the chief star of this triple was suspected by Jacob in 1856 .

Bris. 2199 . This was discovered by Jacob in 1856 .

Lac. 6835. This is not new being Hough 404 . gungen mit.
Innes $I$ in M. N. of R. A. S. Vol. LV. This is not Bris. 18 but UA 64 Toucani, and was noted as a double star at Cordoba in 1873 .

Innes $42=$ Yarn. 9104. In Journal B. Ast. Association Vol. VI April 1896 . With the 7 in. equ. of the Cape Observatory this star is not double. - It will be omitted from further lists.

Innes 18. This was wrongly identified as Lac. 8600 . It is Lac. 8602 , mag. 7.4 , RA. $20^{\mathrm{h}} 47^{\mathrm{m}} 53^{\mathrm{s}}$, S. Decl. $52^{\circ} 29^{\prime} \cdot 7$ (1900); $1896.7: 5^{\circ} \pm 4^{\prime \prime} \pm, 7.5$ and 11.7 .

$K r$.

\title{
Die gegenwärtige Helligkeit der Nova (T) Aurigae.
}

$\mathrm{Da}$ ich am 26. November Gelegenheit hatte, die Nova Aurigae wieder einmal zu photographiren, so benutzte ich dieselbe, um die Nova bezüglich ihrer Helligkeit an Plejadensterne photographisch anzuschliessen.

Ich habe, da die Durchmesser bei der Kürze der Aufnahmezeiten von jeweils 30 Minuten zu klein waren für mikrometrische Messung, den Anschluss durch Stufenschätzen ausgeführt. Aus einer grösseren Anzahl Vergleichungen habe ich die brauchbaren in der folgenden Tabelle zusammengestellt,

\begin{tabular}{|c|c|c|c|c|c|c|c|}
\hline Stern & 70 & $\mathrm{um}$ & 2 & Stufen & heller & als & Nova \\
\hline$»$ & $6 I$ & \# & $I$ & $»$ & $\gg$ & » & » \\
\hline » & 90 & $»$ & 2 & $\gg$ & schwächer & » & $\otimes$ \\
\hline$》$ & 85 & $》$ & 2 & $"$ & heller & $»$ & $"$ \\
\hline$\gg$ & 88 & $\Rightarrow$ & I & $\gg$ & schwächer & $》$ & $\gg$ \\
\hline 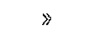 & 92 & $»$ & 0 & $»$ & » & $»$ & $》$ \\
\hline$\gg$ & 80 & $»$ & 2 & $》$ & heller & » & $»$ \\
\hline 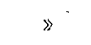 & 74 & $»$ & $\mathbf{I}$ & $»$ & schwächer & » & $»$ \\
\hline » & 93 & » & 2 & $»$ & heller & » & $»$ \\
\hline ४ & 89 & $\gg$ & 0 & $»$ & schwächer & $\gg$ & 》 \\
\hline
\end{tabular}
wobei die Nummern der Sterne diejenigen des Wolf'schen Catalogs, also dieselben wie bei Charlier sind. Es erscheint

Heidelberg, 1896 Dec. 2.
Die Stufe kann ich in diesem Falle als rund $1 / 4$ einer

\begin{tabular}{|c|c|c|}
\hline Stern & $\begin{array}{l}\text { Gr. nach } \\
\text { Charlier }\end{array}$ & $\begin{array}{l}\text { Helligkeit } \\
\text { der Nova }\end{array}$ \\
\hline 70 & I $2{ }^{m} \cdot 5$ & I $3 \div 0$ \\
\hline $6 \mathrm{I}$ & I 2.5 & 12.7 \\
\hline go & 13.5 & 13.0 \\
\hline 85 & 12.5 & 13.0 \\
\hline 88 & I 3.5 & 13.0 \\
\hline 92 & 13.0 & 13.0 \\
\hline 80 & 12.0 & 12.5 \\
\hline 74. & 13.0 & I 2.7 \\
\hline 93 & 12.5 & 13.0 \\
\hline 89 & 13.0 & 13.0 \\
\hline
\end{tabular}
Grössenclasse annehmen, so dass sich für die Nova die folgenden Helligkeiten ergeben:

Hieraus folgt fur die Nova die Helligkeit I $^{\mathrm{m}} \cdot 9$. Man kann also annehmen, dass die Nova Aurigae gegenwärtig (Nov. 26, i 896 ) fast genau von der dreizehnten Grösse bezüglich ihrer photographischen Helligkeit ist. Es ist noch zu bemerken, dass der Stern 6 I gegenwärtig etwas schwächer als der Stern 70 ist, während Charlier und Wolf beide Sterne einander gleich gefunden haben.

Max Wolf.

\section{Bemerkung zu der Abhandlung „Ueber die systematischen Fehler der Distanzmessungen mit neueren Heliometern ", Astr. Nachr. 3397-98.}

In genannter Abhandlung sucht Herr Dr. Cohn den etwaigen constanten Fehler der Heliometermessungen dadurch zu ermitteln, dass er die Hauptdistanz zwischen den Endsternen eines Sternbogens mit der Summe der Partialdistanzen, nachdem letztere auf die Hauptdistanz projicirt sind, vergleicht. Er behandelt dabei diese Projectionen der Partialdistanzen wie direct gegebene Grössen; in Folge dessen glaubt er von den Unsicherheiten der Meridianbestimmungen frei zu werden und die Correctionen der letzteren ableiten zu können. Dies Verfahren ist jedoch nur 
dann erlaubt, wenn die sämmtlichen Sterne eines Bogens $\mathrm{seh} \mathbf{r}$ ahe in einem Bogen grössten Kreises liegen; wenigstens, sobald die Projectionsfactoren aus den Meridianpositionen abgeleitet sind, wie es in den betrachteten Fällen (ausser bei dem Elkin'schen Controlbogen) zutrifft.

Ich habe vor etwa Io Jahren darauf aufmerksam gemacht, dass die üblichen Projectionsfactoren in beträchtlichem Grade von der Unsicherheit der Meridianpositionen abhängig sind, und zwar von der Unsicherheit in einer zur Hauptdistanz senkrechten Richtung. Es sind daher auf meinen Vorschlag die Bd. V S. $3^{62}$ u. 371 der „Venusdurchgänge von 1874 und $1882 \%$ angegebenen Differentialquotienten der Logaritbmen der Projectionsfactoren für Hydra- und Cygnusbogen von mir berechnet worden, dieselben sind erheblich von der Unsicherheit der Rectascensionen abhängig. Ich halte jedoch überhaupt die Einführung der Projectionsfactoren für verwerflich; dieselben scheinen

Berlin I 897 Jan. 20. hauptsächlich dazu zu dienen, um den Einfluss der Unsicher heit der Meridiąnpositionen zu verhullen; Vortheil böten die Projectionsfactoran nur dann, wenn sie aus Positionswinkelmessungen an Heliometern mit genügender Schärfe abgeleitet wären. Man kommt zu dem gleichen Ziel, nämlich der grösstmöglichen Elimination der Oerter der inneren Sterne, wenn man die Summe der heliometrisch bestimmten Distanzen mit der Summe der aus den Meridianbeobachtungen abgeleiteten vergleicht; hierdurch erhält man den nothwendig verbleibenden Einfuss der inneren Sterne in der bequemsten und übersichtlichsten Form. Es hätte dieser Einfluss jedenfalls angegeben werden missen. Ich verweise auf Astr. Nachr. Bd. 122 S. $376 \mathrm{ff}$.

Die Göttinger Controlbogen liegen sehr nahe in einem Bogen grössten Kreises, und es dürfte für diese der betrathtete Einfluss gering sein.

\section{H. Battermann.}

\section{Flecke auf Jupiter.}

Am 29. November vorigen Jahres fand ich am 7 Zöller bei Luft II und Vergr. 233 einen stiefdunklen Fleck in Nord-Aequ.-Band, länglich in der Richtung nach NO.* Derselbe war auch an einem Fraunhofer von $77 \mathrm{~mm}$ und Vergrösserung I 3 I nach der Bemerkung im Tagebuch noch \gut zu sehen $*$. Letzteres Citat möge als Hinweis auf die leichte Sichtbarkeit gelten. Der Fleck, zum kleinen Theil auf dem schmalen und matten Bande liegend, mit dem dünneren Ende in der nördlicheren hellen Zone, schien fast ein Doppelfleck zu sein, aus zwei ungleichen rundlichen Scheibchen bestehend. Nach ziemlich genauen Abmessungen war am 29. November seine Länge $\lambda=74: 5$. Am 3. November hatte ich Jupiter bei Luft II-III, ${ }^{i}$ Vergr. I 76 , gezeichnet, am 10. November ebenfalls bei Luft I-II, Vergrösserung 233 und $\lambda$ war für Jupiter $30^{\circ} 9$ resp. $54^{\circ} 3$. Beide Mal war keine Spur eines dunklen Flecks zu be.

Landstubl, Privatsternwarte, I897 Januar. merken, obwohl die betreffende Gegend sehr gut sichtbar gewesen wäre. Inzwischen hat das Wetter keine Beobachtung mehr erlaubt, doch meldete Herr $W$. Juul aus Kopenhagen eine Wahrnehmung vom 20. Januar I897, am 6 Zöller geschehen, nach welcher in der Breite meines Flecks zwei nahe dunkle Flecke vorhanden wären. Leider liegt lediglich diese Mittheilung vor; jedoch sind fast genau I 25 Rotationen seit meiner Zeichnung verstrichen, weshalb eine Identität zweifellos sein dürfte, mein damals schon eingeschnürter Fleck sich wirklich getheilt hätte und jeder Theil seine eigene Geschwindigkeit besässe.

Vielleicht wiederholt sich, was in der vorigen Opposition bei denr Fleck in der nämlichen Breite beobachtet wurde, weshalb es gestattet sein möge, auf die Verfolgung und die Beobachtung der Meridiandurchgänge des Objects hinzuweisen.

\section{Ph. Fauth.}

\section{Notiz.}

Auf der beigegebenen Reproduction der Lowell'schen Venuskarte (Tafel III) sind leider einige Buchstaben nicht genügend deutlich zum Ausdruck gekommen. Ich bitte daher dieselben an den folgenden Stellen einzeichnen zu wollen.

\begin{tabular}{|c|c|c|c|c|c|c|c|c|c|c|c|}
\hline & $\lambda$ & $\beta$ & & $\lambda$ & $\beta$ & & $\lambda$ & $\beta$ & & $\lambda$ & $\beta$ \\
\hline$a$ & $349^{\circ}$ & $+29^{\circ}$ & $g$ & $61^{\circ}$ & $+22^{\circ}$ & $k$ & $73^{\circ}$ & $-8^{\circ}$ & $s$ & $5^{\circ}$ & $-23^{\circ}$ \\
\hline 6 & $33^{2}$ & -15 & $h$ & I 2 & +17 & $n$ & 2 I & -20 & $u$ & 38 & -7 \\
\hline & 47 & -20 & $j$ & 73 & +7 & 0 & 57 & -44 & $v$ & 5 & +43 \\
\hline
\end{tabular}

\section{Inhalt:}

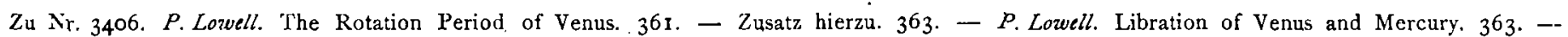
A. E. Douglass. Projections on the Terminator of Mars and Martian Meteorology. 363. - E. C. Pickering. The Bruce Photographic Telescope. 367 . D. Gill. First List of Double Stars. 369 . - Zusatz hierzu. 373. - M. Wolf. Die gegenwärtige Helligkeit der Nova (T) Aurigae. 373. - H. Battermann. Bemerkung zu der Abhandlung »Ueber die systematischen Fehler der Distanzmessungen mit neueren Heliometern «, Astr. Nachr. 3397-98. 373. - Ph. Fauth. Flecke auf Jupiter. 375. - Notiz. 375. 